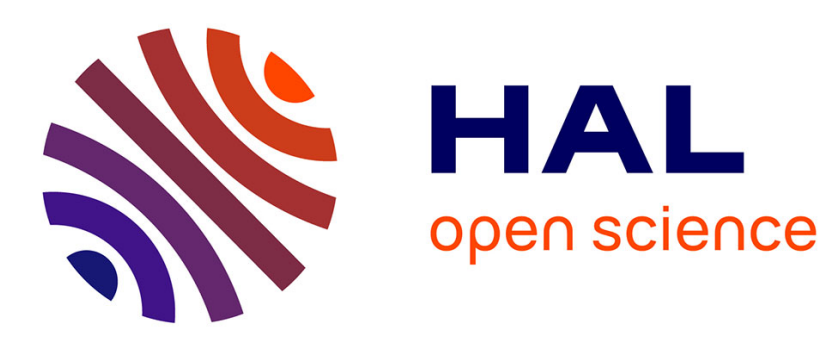

\title{
Comparison between Modelling and Experimental Results of Magnetic Flux trapped in YBCO Bulks
}

Frédéric Trillaud, Kévin Berger, Bruno Douine, Jean Lévêque

\section{To cite this version:}

Frédéric Trillaud, Kévin Berger, Bruno Douine, Jean Lévêque. Comparison between Modelling and Experimental Results of Magnetic Flux trapped in YBCO Bulks. IEEE Transactions on Applied Superconductivity, 2016, 26 (3), pp.6800305. 10.1109/TASC.2016.2518580 . hal-01245842

\section{HAL Id: hal-01245842 \\ https://hal.science/hal-01245842}

Submitted on 12 Jan 2016

HAL is a multi-disciplinary open access archive for the deposit and dissemination of scientific research documents, whether they are published or not. The documents may come from teaching and research institutions in France or abroad, or from public or private research centers.
L'archive ouverte pluridisciplinaire $\mathbf{H A L}$, est destinée au dépôt et à la diffusion de documents scientifiques de niveau recherche, publiés ou non, émanant des établissements d'enseignement et de recherche français ou étrangers, des laboratoires publics ou privés. 


\title{
Comparison between Modelling and Experimental Results of Magnetic Flux trapped in YBCO Bulks
}

\author{
Frederic Trillaud, Kévin Berger, Bruno Douine and Jean Lévêque
}

\begin{abstract}
An electromagnetic simulation of YBCO bulks was performed and the resulting trapped magnetic flux density was compared to Field Cooling experimental measurements for an applied magnetic flux density up to $3 \mathrm{~T}$. The simulation relied on an axisymmetric problem implementing an $A$-formulation of the Maxwell's equations solved by means of the Finite Element Method so that the time evolution of the magnetic flux density was computed over the cross section of the bulk. To derive its electrical conductivity, a classic power law was adopted that includes the dependence of the critical current density upon temperature and external magnetic field modelled on the basis of a Modified Kim-Anderson relation. It was found that this model tends asymptotically towards the critical state for which the $n$-value becomes a free parameter that should be then estimated experimentally. The experimental results could be fairly reproduced at different operating temperatures with the best trapping at temperatures below $77 \mathrm{~K}$ benefiting from an increasing critical current density.
\end{abstract}

Index Terms-Modified Kim-Anderson relation, trapped magnetic flux, YBCO bulk modelling.

\section{INTRODUCTION}

$\mathbf{T}$ ARGETING practical applications of High-Temperature Superconducting (HTS) bulks [1]-[3], a great deal of effort has been carried out regarding the art of growing materials, their properties and their modelling [4]-[7]. With regards to the latter, analytical formulae, in the simplest 2D cases using the Bean's Critical State Model (BCSM), and numerical models, in the most complex geometries involving 3D modelling techniques and/or more refined critical current dependencies, have been developed to predict the magnitude of the penetration field and the feasibility of trapping large magnetic fluxes [8]-[10]. The present work is an incremental step in the field of HTS bulk modelling with the objective of reproducing experimental data and predicting the electromagnetic behaviour of disk-shaped samples. To this end, the Finite Element Method (FEM) was used and a Modified KimAnderson (MKA) relation was implemented to describe the dependence of the current density on the temperature and the background magnetic flux density.

The study is carried out under the main assumptions that the bulk and the background electromagnet are isotropic and homogeneous solids with cylindrical symmetry, reducing

F. Trillaud is with the Institute of Engineering, National Autonomous University of Mexico, D.F., 04510 Mexico (e-mail: ftrillaudp@pumas.ii.unam.mx)

K. Berger, B. Douine and J. Lévêque are with the University of Lorraine, GREEN, Research Group in Electrical Engineering and Electronics of Nancy - EA 4366, Faculté des Sciences et Technologies, BP 70239, 54506 Vandoeuvre-lès-Nancy Cedex, France.

Final manuscript received January 12, 2016. the complexity of a 3D problem to a simpler axisymmetric one. The Faraday's law of induction and the Ampère's law were lumped into a single electromagnetic equation via the magnetic vector potential $\mathbf{A}$. In this $A$-formulation, the electrical conductivity of the sample was expressed through the classic power law describing the $E-J$ characteristics of HTS samples. However, to be able to reconcile this particular formulation with an axisymmetrical model, the induced current is assumed to be solely arising from the component of the magnetic field along the axis of symmetry [11]. The resulting mathematical expression was extremized into a weak form. Subsequently, the solution was computed using the Galerkin method [12] built into the free solver GetDP of the open source software Gmsh [13], [14]. The function space is decomposed on the basis of first-order edge elements [15] and a Backward Differentiation Formula (BDF) method with an adaptive time step scheme allowed integrating the solution over space and time.

In this publication, the experimental setup used to magnetize YBCO samples under Field Cooling (FC) conditions is briefly presented followed by a detailed description of the electromagnetic model. Finally, numerical results are compared to experimental data. It is then inferred that the representation of the critical surface through the MKA relation can approximate with high degree of accuracy the FC magnetization of YBCO bulks for a background magnetic flux density of up to $3 \mathrm{~T}$.

\section{NUMERICAL ANALYSIS}

\section{A. Experiment setup and its modelling}

A disk-shaped YBCO sample, prepared following a top seeded melt growth process, was placed at the centre of a $\mathrm{LHe}$ cooled NbTi background electromagnet that was connected to a current-regulated DC power supply [5]. The sample was encapsulated in a copper jacket which was anchored to a 2stage cryocooler to be cooled down to the desired operating temperature. In this set up, FC experiments were conducted at 12,30 and $77 \mathrm{~K}$ on a single sample. The background magnetic field was first ramped up while the sample was in its normalresistive state. Once the background magnetic field reached a plateau at its nominal value, the sample was cooled to the desired operating temperature. Then, the background magnetic field was ramped down to zero at a rate of approximately $0.003 \mathrm{~T} / \mathrm{s}$ so as to magnetize the sample. A Hall sensor was placed at the top centre surface of the bulk to record the time evolution of the magnetic flux density. Further details of the experiment and its procedure can be found in [16]. At a rate of $0.003 \mathrm{~T} / \mathrm{s}$, the local dissipation is expected to be negligible. 


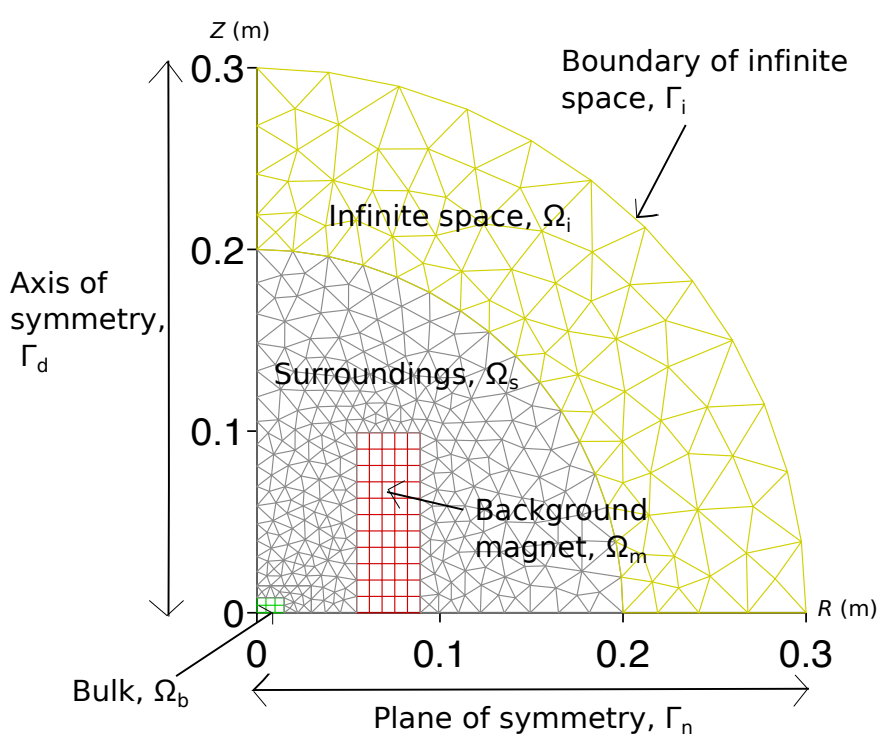

Fig. 1. Model and mesh used to compute the background $B_{\text {a }}$ and measured magnetic flux density $B_{\mathrm{m}}$ over $1 / 4^{\text {th. }}$ of the cross section of the sample. The dimensions of the background magnet and sample can be found in Table I

Hence the temperature is assumed to be constant throughout the magnetization process [16].

A coarser mesh of the geometrical model than the one used to compute the solution is shown in Fig. 1. It is implicitly assumed that the problem is perfectly axisymmetric with homogeneous and isotropic material properties. The model is divided in 4 regions representing $1 / 4^{\text {th. }}$ of the experimental set up: $\Omega_{\mathrm{m}}$ for the background electromagnet, $\Omega_{\mathrm{b}}$ for the sample, $\Omega_{\mathrm{s}}$ for the close surroundings of both electromagnet and bulk, and an additional $\Omega_{\mathrm{i}}$ region to simulate the diffusion and closing of the magnetic field in the infinite non-magnetic space surrounding the whole experiment. Table I summarizes the geometrical dimensions of the studied sample and the background electromagnet. The Ampère-turns of the electromagnet were computed to match the measured intensity of the applied magnetic flux density.

\section{B. Electromagnetic model: A-formulation}

Finite Element Analysis (FEA) is proposed here to study the magnetization of YBCO bulks introducing a detailed $J_{\mathrm{c}}(T, B)$ model. Amongst the body of available formulations, the $A$ formulation was chosen for its straightforward implementation into the solver GetDP. The basis of the model is the divergence free property of the magnetic flux density, allowing one to introduce the magnetic vector potential $\mathbf{A}$. Rewriting the Maxwell's equations in terms of $\mathbf{A}$ leads to the general expression given below

$$
\nabla \times\left[\frac{1}{\mu_{0}} \nabla \times \mathbf{A}\right]+\sigma \frac{\partial \mathbf{A}}{\partial t}-\mathbf{J}_{\mathbf{a}}=0,
$$

where $\mathbf{J}_{\mathrm{a}}$ is the imposed engineering current density flowing through the background electromagnet and $\mu_{0}$ is the permeability of vacuum. The expression of the electrical conductivity of the bulk $\sigma$ is given by the power law expression

$$
\sigma=J_{\mathrm{c}} E_{\mathrm{c}}^{-\frac{1}{n}}\left(\left\|\frac{\partial \mathbf{A}}{\partial t}\right\|+\epsilon\right)^{\frac{1-n}{n}}+\frac{1}{\rho_{\mathrm{nsc}}} .
$$

where $J_{\mathrm{c}}, E_{\mathrm{c}}, n$ and $\rho_{\text {nsc }}$ are the critical current density, the critical electrical field, the $n$-value and the normal-state resistivity of the bulk, respectively. The introduction of $\epsilon=$ $10^{-6} E_{\mathrm{c}}$ in expression (2) avoids any divisions by zero when $\partial \mathbf{A} / \partial t=0$. The normal-state resistivity $\rho_{\text {nsc }}$ was obtained from film data found in [17] and fitted to the following equation

$$
\rho_{\text {nsc }}(T)=10^{-6} \times(1.6+0.012 \times T),
$$

where $T$ is the operating temperature. The presence of the normal-state resistivity allows one to simulate the smooth transition between the normal-state resistance above the critical temperature to zero resistance in the superconducting state during FC simulations, which also improves the numerical convergence [18].

Following a sensitivity analysis, it was noted that the magnitude of the trapped magnetic flux density upon increasing the $n$-values was decreasing until no significant changes could be observed. Indeed, according to (2) and neglecting the numerical terms $\epsilon$ and the normal-state resistivity $\rho_{\text {nsc }}$, as the $n$-value gets larger and larger, the electrical conductivity becomes independent of $n$, and

$$
\sigma \underset{n \rightarrow \infty}{\sim} \frac{J_{\mathrm{c}}(T, B)}{\left\|\frac{\partial \mathbf{A}}{\partial t}\right\|} .
$$

For the following studies, $n$-values were computed on the basis of the expression provided in [19]: 30 at $77 \mathrm{~K}, 60$ at $30 \mathrm{~K}, 180$ at $12 \mathrm{~K}$, at which values the trapped magnetic flux density is found insensitive. At a temperature of $77 \mathrm{~K}$, for which measurements are available, the expected $n$-values for HTS bulks range from 18 to 45 according to [20]-[22], being consistent with the proposed value.

It should be noted that the mathematical problem is correctly stated as long as the applied magnetic flux density induces a current that can follow the electric field without encountering any boundaries in its path [11]. It is such when the bulk is an isotropic solid with cylindrical symmetry, subjected to an applied magnetic field along the vertical axis. Hence, the current flows freely as concentric circles around the axis of symmetry and (1) is sufficient to describe the electromagnetic behaviour of the system without the necessity of introducing the electric scalar potential $V$.

Finally, to simulate the infinite non-magnetic space surrounding the experimental set up, a shell transformation is applied to the outer rim $\Omega_{\mathrm{i}}$ as described in [23]. Dirichlet and Neumann boundary conditions are applied at the edges of the model (Dirichlet: $\Gamma_{\mathrm{i}}$ and $\Gamma_{\mathrm{d}}$, Neumann: $\Gamma_{\mathrm{n}}$, see Fig. 1).

\section{MOdIFIED KIM-ANDERSON RELATION}

The Kim-Anderson relation is a widely used model to express the dependence of the critical current density upon magnetic flux density and temperature [24]-[26]. To reproduce 
TABLE I

DIMENSIONS OF BULK AND BACKGROUND ELECTROMAGNET.

\begin{tabular}{|c|c|}
\hline Parameters & Values $(\mathrm{cm})$ \\
\hline \multicolumn{2}{|c|}{ Background electromagnet } \\
\hline Inner radius & 5.5 \\
\hline Outer radius & 9 \\
\hline Height & 20 \\
\hline \multicolumn{2}{|c|}{ Bulk sample } \\
\hline Radius & 1.5 \\
\hline Thickness & 1.67 \\
\hline
\end{tabular}

the experimental data over a broad range of experimental temperatures and background magnetic flux densities, the relation was altered to give the following modified version [27], [28]

$$
J_{\mathrm{c}}(T, B)=\frac{J_{\mathrm{c} 0}(T)}{\left(1+\frac{B}{B_{0}(T)}\right)^{\alpha}},
$$

where $J_{\mathrm{c} 0}$ is the temperature-dependent critical current density at zero magnetic field and $\alpha(=0.7)$ is a dimensionless constant that yields the Bean's critical state function when equal to 0 and the Kim-Anderson relation when equal to 1 [29]. $B$ is the norm of the applied magnetic flux density and $B_{0}$ is a macroscopic normalising parameter whose temperature dependence was fitted to

$$
B_{0}(T)=B_{00} \times\left[1-\delta\left(\frac{T}{T_{\mathrm{c} 0}}\right)^{\beta}\right],
$$

where $B_{00}, \delta$ and $\beta$ are fit parameters, and $T_{\mathrm{c} 0}=92 \mathrm{~K}$ is the critical temperature. The critical current density $J_{\mathrm{c} 0}$ was extrapolated to $0 \mathrm{~T}$ following a polynomial equation on the basis of a linear model presented in [19]

$$
J_{\mathrm{c} 0}(T)=J_{\mathrm{c} 00} \times\left(\frac{T-T_{\mathrm{c} 0}}{T_{\mathrm{ref}}-T_{\mathrm{c} 0}}\right)^{\gamma},
$$

where $\gamma$ is a fit parameter. The best parameters to match the numerical and experimental results over the different operating temperatures (see Fig. 3) were found to be: $B_{00}=2.67 \mathrm{~T}$, $\delta=1.54, \beta=2.56, J_{\mathrm{c} 00}=J_{\mathrm{c} 0}\left(T_{\text {ref }}\right)=109 \mathrm{~A} / \mathrm{mm}^{2}$ with $T_{\text {ref }}=77 \mathrm{~K}$ and $\gamma=0.96$. To get a sense of the viability of the chosen model, Fig 2 compares the proposed current density model with experimental data published in [30], [31] for a YBCO bulk having $J_{\mathrm{c}} \simeq 350 \mathrm{~A} / \mathrm{cm}^{2}$ at $B \simeq 0 \mathrm{~T}$ and $77 \mathrm{~K}$ against values currently obtained at the authors' university laboratory in the range of $100-200 \mathrm{~A} / \mathrm{cm}^{2}$ [10], [32]. The former data corresponds to a magnetic flux density applied along the c-axis. Bearing in mind that the critical surface is a unique characteristic of a given superconducting sample, and that the direction of the applied magnetic field on the sample typically affects its characteristics, values of the critical current density and its general dependence on the background magnetic flux density are considered within acceptable range of sample-to-sample variabilities in quality. Additionally, it was possible to fit the experimental data of [30] up to $2 \mathrm{~T}$ at a temperature below $77 \mathrm{~K}$ and up to $3 \mathrm{~T}$ at a temperature

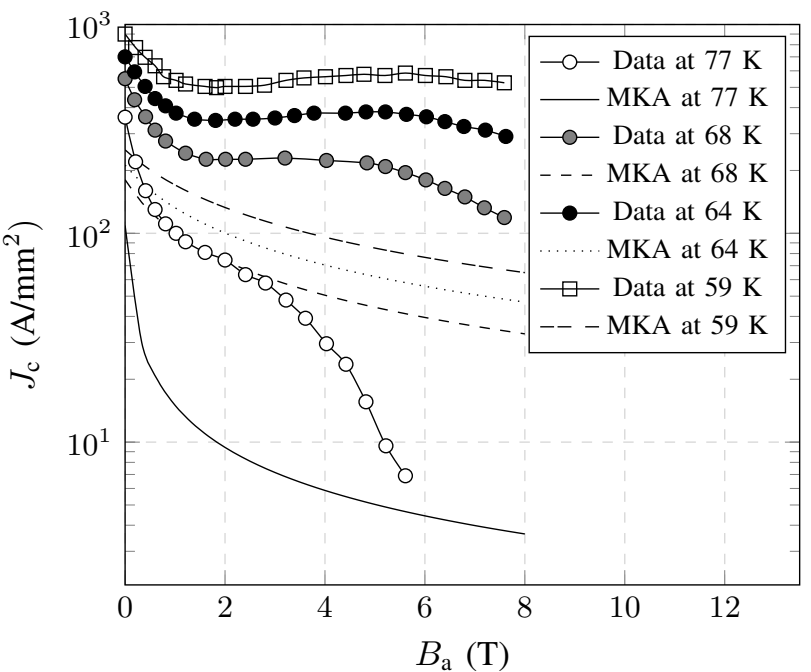

Fig. 2. Evolution of critical current density of a YBCO bulk at different operating temperatures as a function of an external magnetic flux density applied parallel to the c-axis compared to values obtained from the MKA relation (5). These values even though lower than ones found in literature remain within the range of acceptable sample-to-sample variability arising from the manufacturing process.

of $77 \mathrm{~K}$ with the appropriate set of parameters of the MKA relation which gives one strong key point to warrant the relevancy of the chosen $J_{\mathrm{c}}(T, B)$ relation for the mentioned range of operating values. Nevertheless, the proposed model is an idealization of bulk characteristics with a limited set of parameters that can only depict a smooth dependency upon the applied magnetic flux density. More refined models should be considered to explore regions beyond $3 \mathrm{~T}$.

\section{COMPARISON BETWEEN EXPERIMENTAL AND NUMERICAL RESULTS}

Fig. 3, 4 and 5 show the different experimental measurements obtained on the bulk top centre surface and their corresponding simulations. The measured magnetic flux density $B_{\mathrm{m}}$ was computed using the MKA relation given by (5) and the induced magnetic flux density $B_{\mathrm{i}}$ was derived by means of

$$
B_{\mathrm{i}}=B_{\mathrm{a}}-B_{\mathrm{m}},
$$

where $B_{\mathrm{a}}$ is the norm of the background magnetic flux density. Once the background field has vanished, a fraction of the magnetic flux density will remain trapped. The largest trapped magnetic flux densities were obtained at $12 \mathrm{~K}$ and $30 \mathrm{~K}$, amounting to nearly $77 \%$ and $68 \%$ of the nominal background magnetic flux density, respectively. However, at $77 \mathrm{~K}$, only $10 \%$ of the nominal applied magnetic flux density was trapped. In terms of the simulation, the proposed model fairly reproduced the behaviour of measured magnetic flux densities as well as the magnitude of the corresponding trapped magnetic flux densities throughout the different operating temperatures. At $77 \mathrm{~K}$, the matching is nearly perfect whereas the computed curves at 12 and $30 \mathrm{~K}$ show a miscellaneous discrepancy during the decrease of the background magnetic field with a 


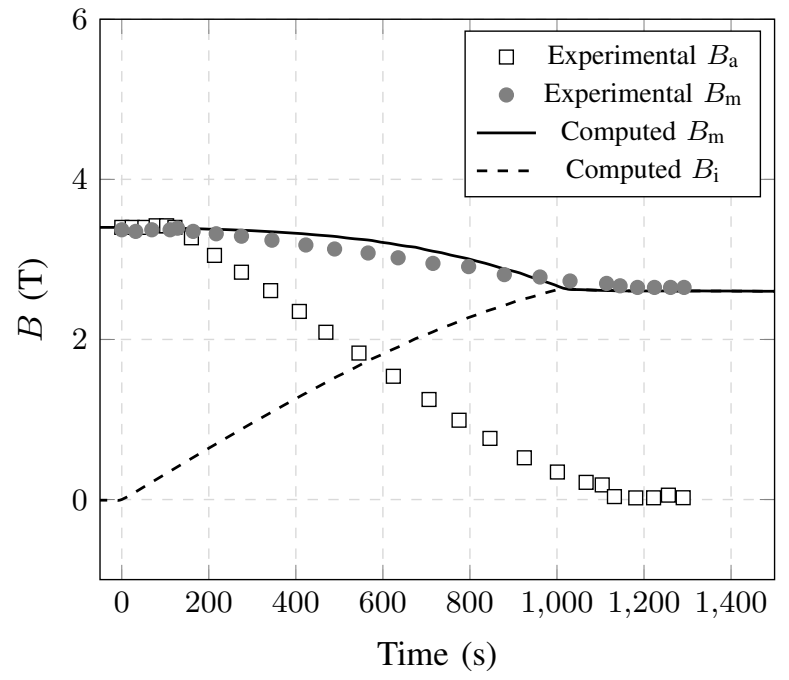

Fig. 3. Time evolution of the magnetic flux density at the top centre surface of the sample at $12 \mathrm{~K}$ and its comparison with simulations. The square " $\square$ " and filled bullet "•" markers indicate the time evolution of the background magnetic flux density $B_{\mathrm{a}}$ and measured magnetic flux density $B_{\mathrm{r}}$, respectively. The solid "-" and dashed "- _" lines correspond to numerical simulations of the measured magnetic flux density $B_{\mathrm{m}}$ and the magnetic flux density $B_{\mathrm{i}}$.

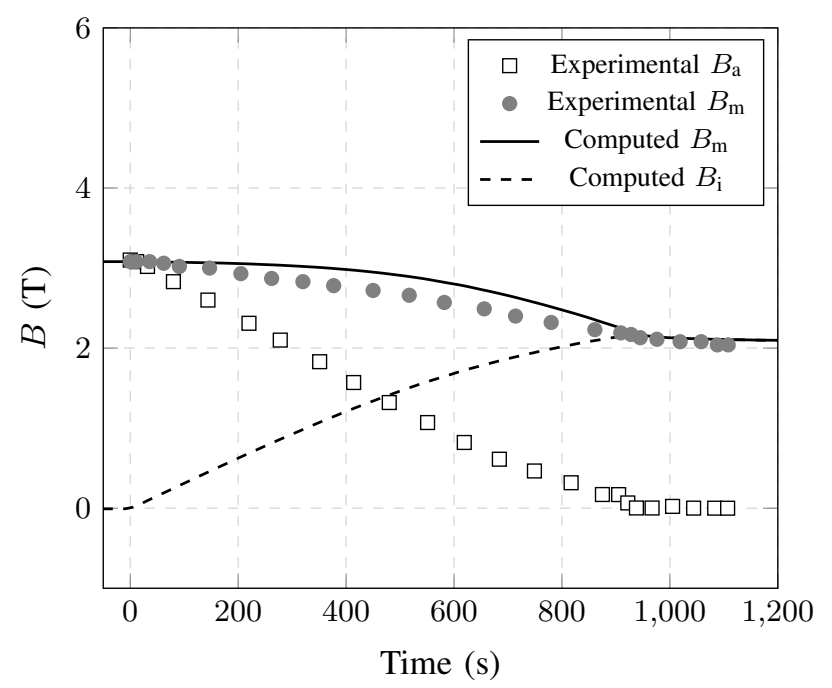

Fig. 4. Time evolution of the magnetic flux density at the top centre surface of the sample at $30 \mathrm{~K}$ and simulations.

slightly rounder profile. This discrepancy ought to be related to the MKA relation describing $J_{\mathrm{c}}(T, B)$ and the rate of change of the magnetic vector potential according to (4).

\section{Discussion}

The magnetization curves are mostly shaped by the evolution of the local electrical resistance of the bulk; the latter being fully defined by the parameters of the MKA relation and the rate of change of the background magnetic field. These parameters mainly depend on the manufacturing process and the composition of the sample. As mentioned previously, the $n$-value had barely any influences on the results in the range of expected values which are typically several tens as reported in the literature. It becomes a free parameter that

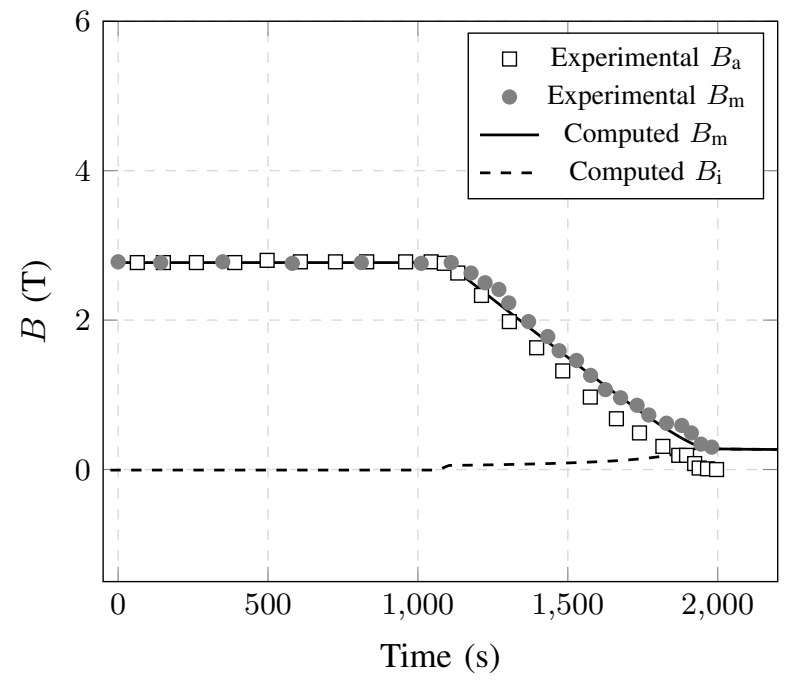

Fig. 5. Time evolution of the magnetic flux density at the top centre surface of the sample at $77 \mathrm{~K}$ and simulations.

cannot be inferred from the numerical model and should be measured experimentally. As a result, the proposed $E-J$ characteristic reaches asymptotically towards a critical state model. The parameters shaping the results are then contained in the dependence of the critical current density upon magnetic flux density and temperature for a fixed rate of change of magnetic field. Thus, increasing $J_{\mathrm{c} 0}$ and $B_{0}$ allows trapping more flux with a lower slope as the background magnetic field vanishes. These parameters as they are risen up modify the critical surface such that larger values of $J_{\mathrm{c} 0}(T)$ are achieved and the curves $J_{\mathrm{c}}(T, B)$ show a lesser dependence upon background magnetic flux density than the ones presented in Fig. 2. As more data are available, it would be possible to reconstruct with greater accuracy the critical surface up to $3 \mathrm{~T}$. Beyond this value, a more refined relation may be more appropriate and alternative fitting curves with additional independent parameters might be tried out.

\section{CONCLUSION}

The introduction of the MKA relation into the power law allowed reproducing fairly accurately the experimental data at temperatures of 12,30 and $77 \mathrm{~K}$ in FC conditions up to $3 \mathrm{~T}$. Reaching towards the critical state, the $n$-value becomes a free parameter whose values can only be acquired through experimental measurements. Then, the best trapping was achieved at temperatures lower than $77 \mathrm{~K}$ owing to an increasing critical current density. For larger applied magnetic flux densities, it is suggested to explore alternative $J_{\mathrm{c}}(T, B)$ models with greater degrees of parametrization in order to be able to fit the critical surface beyond $3 \mathrm{~T}$. One possibility is the reconstruction of the electrical resistivity as a function of the background magnetic flux density for a given operating temperature and from there one would derive the corresponding critical surface. This alternative approach would allow one to validate various assumptions including the power law and the $J_{\mathrm{c}}(T, B)$ models taking into account sample-to-sample discrepancies resulting from preparation and history. 


\section{REFERENCES}

[1] L.K. Kovalev, K.V. Ilushin, V.T. Penkin, K.L. Kovalev, A.E. Larionoff, S.M-A. Koneev, K.A. Modestov, S.A. Larionoff, V.N. Poltavets, I.I. Akimov, V.V. Alexandrov, W. Gawalek, B. Oswald, and G. Krabbes, "High output power reluctance electric motors with bulk high-temperature superconductor elements," Superconducting Science and Technology, vol. 15 , pp. $817-822,2002$.

[2] G. Malé, T. Lubin, , S. Mezani, J. Lévêque, "Analytical calculation of the flux density distribution in a superconducting reluctance machine with HTS bulks rotor," Mathematics and Computers in Simulation, vol. 90, pp. 230-243, April 2013. ELECTRIMACS 2011- PART I.

[3] M. Miryala, "Superconductivity: Applications Today and Tomorrow," Nova Science Publishers, Inc., 2016.

[4] S.B. Yan, Y.Y. Chen, H. Ikuta, and X. Yao, "Enhanced Growth Rate of a YBCO bulk in the Melt-Textured Process Under 1 atm Oxygen Pressure," IEEE Transactions on Applied Superconductivity, vol. 20, pp. 66-70, April 2010.

[5] W. Zhai, Y. Shi, J.H. Durrell, A.R. Dennis, Z. Zhang, and D.A. Cardwell, "Processing and Properties of Bulk YBaCuO Superconductors Fabricated by Top Seeded Melt Growth from Precursor Pellets Containing a Graded $\mathrm{CeO}_{2}$ Composition," Crystal Growth \& Design, vol. 15, pp. 907-914, 2015.

[6] Y. Feng, J.G. Wen, A.K. Pradhan, N. Koshizuka, L. Zhou, S.K. Chen, K.G. Wang, and X.Z. Wu, "Preparation and properties of PMP YBCO bulk with submicrometre $\mathrm{Y}_{2} \mathrm{BaCuO}_{5}$ particles," Superconductor Science Technology, vol. 13, pp. 703-708, 2000.

[7] Z. Hong, Ph. Vanderbemden, R. Pei, Y. Jiang, A. M. Campbell, and T. A. Coombs, "The Numerical Modeling and Measurement of Demagnetization Effect in Bulk YBCO Superconductors Subjected to Transverse Field," IEEE Transactions on Applied Superconductivity, vol. 18, pp. 1561-1564, June 2008

[8] E.H. Brandt, "Superconductor disks and cylinder in an axial magnetic field, field penetration and magnetization Curves," Physical Review B, vol. 58, pp. 6506-6522, September 1998.

[9] G.P. Lousberg, M. Ausloos, C. Geuzaine, P. Dular, P. Vanderbemden, B. Vanderheyden, "Numerical simulation of the magnetization of hightemperature superconductors: a 3D finite element method using a single time step Iteration," Superconductor Science and Technology, vol. 22, 2009. 055005

[10] B. Douine, C.-H. Bonnard, F. Sirois, K. Berger, A. Kameni, J. Lévêque, "Determination of $j_{\mathrm{c}}$ and $n$-value of hts pellets by measurement and simulation of magnetic field penetration," IEEE Transactions on Applied Superconductivity, vol. 25, August 2015. 8001008.

[11] A.M. Campbell, "An Introducton to Numerical Methods in Superconductors," Journal of Superconductivity and Novel Magnetism, vol. 24, pp. 27-33, 2011.

[12] A. Kameni, M. Boubekeur, L. Alloui, F. Bouillault, J. Lambretchs, C. Geuzaine, "A 3-D Semi-implicit Method for Computing the Current Density in Bulk Superconductors," IEEE Transactions on Applied Superconductivity, vol. 50, February 2014. 7009204.

[13] Patrick Dular and Christophe Geuzaine, "GetDP: a General Environment for the Treatment of Discrete Problems." [Online]. Available: http://geuz.org/getdp/.

[14] C. Geuzaine and J.-F. Remacle, "Gmsh: a three-dimensional finite element mesh generator with built-in pre- and post-processing facilities." [Online]. Available: http://geuz.org/gmsh/.

[15] R. Brambilla, F. Grilli and L. Martini, "Development of an edge-element model for AC loss computation of high-temperature superconductors," Superconductor Science and Technology, vol. 20, pp. 16-24, 2007.

[16] B. Douine, F. Sirois, J. Leveque, K. Berger, C.-H. Bonnard, T.-C. Hoang, and S. Mezan, "A New Direct Magnetic Method for Determining $\mathrm{J}_{\mathrm{c}}$ in Bulk Superconductors From Magnetic Field Diffusion Measurements," IEEE Transactions on Applied Superconductivity, vol. 22, June 2012. 9001604.

[17] B. Wuyts, V.V. Moshchalkov, and Y. Bruynseraede, "Resistivity and Hall effect of metallic oxygen-deficient $\mathrm{YBa}_{2} \mathrm{Cu}_{3} \mathrm{O}_{x}$ films in the normal state," Physical Review B, vol. 53, pp. 9418-9432, April 1996.

[18] F. Grilli, S. Stavrev, Y.L. Floch, M. Costa-Bouzo, E. Vinot, I. Klutsch, G. Meunier, P. Tixador, B. Dutoit, "Finite-Element Method Modeling of Superconductors: From 2-D to 3-D," IEEE Transactions on Applied Superconductivity, vol. 15, pp. 17-25, March 2005.

[19] K. Berger, J. Lévêque, D. Netter, B. Douine, A. Rezzoug, "Influence of Temperature and/or Field DDependence of the E-J Power Law on Trapped Magnetic Field in bulk YBaCuO," IEEE Transactions on Applied Superconductivity, no. 2, pp. 3028-3031, 2007.
[20] H. Yamasaki and Y. Mawatari, "Current-voltage characteristics and flux creep in melt-textured $\mathrm{YBa}_{2} \mathrm{Cu}_{3} \mathrm{O}_{7}$," Superconducting Science and Technology, vol. 13, pp. 202-208, 2000.

[21] Ph. Vanderbemden, Z. Hong, T.A. Coombs, S. Denis, M. Ausloos, J. Schwartz, I. B. Rutel, N. Hari Babu, D. A. Cardwell, and A. M. Campbell, "Behavior of bulk high-temperature superconductors of finite thickness subjected to crossed magnetic fields: Experiment and model," Physical Review B, vol. 75, p. 174515, 2007.

[22] M.P. Philippe, M.D. Ainslie, L. Wéra, J.-F. Fagnard, A.R. Dennis, Y.-H. Shi, D.A. Cardwell, B. Vanderheyden and P. Vanderbemden, "Influence of soft ferromagnetic sections on the magnetic flux density profile of a large grain, bulk $\mathrm{YBaCuO}$ superconductor," Superconducting Science and Technology, vol. 28, p. 095008 (13pp), 2015.

[23] F. Henrotte, B. Meys, H. Hedia, P. Dular, W. Legros, "Finite Element Modelling with Transformation techniques," IEEE Transactions on Magnetics, vol. 35, pp. 1434-1437, May 1999.

[24] Y.B. Kim, C.F. Hempstead, A.R. Strnad, "Critical Persistent Current in Hard Superconductors," Physical Review Letters, vol. 9, no. 7, pp. 306309, 1962.

[25] P.W. Anderson, "Theory of the flux creep in hard superconductors," Physical Review Letters, vol. 9, no. 7, pp. 309-311, 1962.

[26] P.W. Anderson and Y.B. Kim, "Hard superconductivity: Theory of motion of abrikosov flux lines," Reviews of Modern Physics, vol. 36, January 1964.

[27] Z. Koziol/, J.J.M. Franse, P.F. de Châtel, and A.A. Menovsky, "Magnetization of a superconductor: Results from the critical-state model," Physical Review B, vol. 50, pp. 15978-15992, December 1994.

[28] F. Inanir, S. Yildiz, K. Ozturk, S. Celebi, "Magnetisation of Gd diffused $\mathrm{YBa}_{2} \mathrm{Cu}_{3} \mathrm{O}_{7}$ superconductor: Experiment and theory," Chinese Physics $B$, vol. 22, no. 7, p. $077402,2013$.

[29] S. Tochihara, K.-I. Harashima, H. Yasuoka, H. Mazaki, M. Osada, M. Kakihana, "Temperature Dependence of Lower Critical Fields in a single-Crystal $\mathrm{Bi}_{2} \mathrm{Sr}_{2} \mathrm{CaCu}_{2} \mathrm{O}_{\delta+d}$ Bulk Superconductor," in Advances in Superconductivity X: Proceedings of the 10th. International Symposium on Superconductivity (ISS'97) (K. Osamura and I. Hirabayashi, ed.), vol. 2, pp. 63-66, Springer, October 1997. ISBN: 978-4-431-66881-

[30] G. Krabbles, G. Fuchs, W.-R. Canders, H. May, R. Palka, High Temperature Superconductor Bulk Materials: Fundamental, Processing, Properties Control, Application Aspects. Wiley-VCH, 2006. ISBN: 9783-527-40383-7.

[31] P. Seidel, "Applied Superconductivity: Handbook on Devices and Applications," vol. 2, Wiley-VCH, 2015.

[32] B. Douine, G. Male, T. Lubin, S. Mezani, J. Leveque, K. Berger, "Improvement of YBCO Superconductor Magnetic Shielding by Using Multiple Bulks," Journal of Superconductivity and Novel Magnetism, vol. 27, pp. 903-907, 2014. 